\title{
ON CERTAIN CONTRACTION MAPPINGS IN A PARTIALLY ORDERED VECTOR SPACE ${ }^{1}$
}

\author{
A. C. THOMPSON
}

G. Birkhoff [1] and H. Samelson [4] have shown that a means of solving problems concerning the existence and uniqueness of eigenvectors of positive operators is given by introducing a suitable metric on a subset of the cone with respect to which the operators are contractions. More specifically, they have proved the Perron theorem for matrices with positive elements by intersecting the positive quadrant with a hyperplane and by using the Hilbert metric (see, for example, [3]) on this section. Birkhoff was able to extend this method (using the same metric) to certain positive linear operators in a more general setting. Since the contraction mapping principle is essentially nonlinear it seemed likely that this method could be used for a class of nonlinear operators. In this paper we use a slightly different distance function the domain of definition of which is not restricted to such a section of the cone and we obtain a theorem for a class of nonlinear mappings which contract this metric.

After giving necessary preliminaries the metric is defined in $\$ 2$ and the completeness of certain subsets is proved. This is followed by a theorem on nonlinear operators and two examples.

1. Preliminaries. Throughout this paper $X$ will denote a real, normed, linear space partially ordered by means of a positive cone $K$, that is to say $K$ is a nonempty, closed subset of $X$ with the following properties:

(i) $x, y \in K \Rightarrow x+y \in K$,

(ii) $x \in K, \alpha \geqq 0 \Rightarrow \alpha x \in K$,

(iii) $x \in K,-x \in K \Rightarrow x=0$

and we write $x \leqq y$ if and only if $y-x \in K$. $K$ is said to be normal if there exists a positive constant $\gamma$ such that $0 \leqq x \leqq y$ implies that $\|x\| \leqq \gamma\|y\|$.

LEMMA 1. If $K$ is normal with constant $\gamma$ then $x \leqq \lambda y, y \leqq \lambda x,\|x\| \leqq m$, $\|y\| \leqq m$ together imply $\|x-y\| \leqq m(1+2 \gamma)(\lambda-1)$.

Received by the editors March 28, 1962.

1 This paper consists of part of the work done for a doctoral thesis at King's College, Newcastle upon Tyne. 
Proof. We have $x-y \leqq(\lambda-1) y$ and $y-x \leqq(\lambda-1) x$ hence there exist $z, z^{\prime} \in K$ such that

$$
x-y+z=(\lambda-1) y \text { and } y-x+z^{\prime}=(\lambda-1) x .
$$

Then

$$
\begin{aligned}
\|z\| \leqq \gamma\left\|z+z^{\prime}\right\| & =\gamma\left\|x-y+z+y-x+z^{\prime}\right\| \\
& =\gamma\|(\lambda-1) y+(\lambda-1) x\| \\
& \leqq 2 m \gamma(\lambda-1) .
\end{aligned}
$$

Hence

$$
\begin{aligned}
\|x-y\| & =\|x-y+z-z\| \\
& \leqq\|x-y+z\|+\|z\| \\
& \leqq(\lambda-1) m(1+2 \gamma) .
\end{aligned}
$$

Elements $x$ and $y$ belonging to $K$ but not both zero are said to be linked if and only if there exist finite (positive) real numbers $\lambda$ and $\mu$ with $x \leqq \lambda y$ and $y \leqq \mu x$. This is an equivalence relation which splits $K$ into a set of mutually exclusive constituents (the equivalence classes) each of which is a "blunted" sub-cone, i.e., a subset of $K$ with the properties (1) except that 0 does not belong and they are not closed. In $R^{3}$, for example, with $K$ taken to be the positive quadrant, the constituents are the interior of $K$, the interiors of the three faces of $K$ and the three edges of $K$ (without the origin).

2. The definition of an order metric. Let $x$ and $y$ be linked. Define $\alpha$ and $\beta$ by the equations

$$
\alpha=\inf \{\lambda: x \leqq \lambda y\}, \quad \beta=\inf \{\mu: y \leqq \mu x\},
$$

then, since $K$ is closed, $x \leqq \alpha y$ and $y \leqq \beta x$ so that if either $\alpha=0$ or $\beta=0$ then $x=y=0$ which is excluded since $x$ and $y$ are linked. Let $d(x, y)=\log \{\max (\alpha, \beta)\}$.

Lemma 2. $d($,$) defines a metric on each constituent of K$.

Proof. That $d(x, y)=d(y, x)$ is evident from the definition. If both $x \leqq y$ and $y \leqq x$ then, by property (1) (iii) of $K, x=y$ so that, if $x \neq y$, either $\alpha$ or $\beta$ is strictly greater than 1 . Thus $d(x, y) \geqq 0$ and $d(x, y)=0$ if and only if $x=y$. Finally, suppose $x, y$ and $z$ belong to a constituent and

$$
\begin{array}{ll}
x \leqq \alpha_{1} y, & y \leqq \beta_{1} x \\
x \leqq \alpha_{2} z, & z \leqq \beta_{2} x \\
z \leqq \alpha_{3} y, & y \leqq \beta_{3} z
\end{array}
$$


where $\alpha_{i}, \beta_{i}$ are the infima of all scalars satisfying the respective inequalities. Then, if $\alpha_{1} \geqq \beta_{1}$,

$$
\begin{aligned}
d(x, y) & =\log \alpha_{1} \leqq \log \left(\alpha_{2} \alpha_{3}\right)=\log \alpha_{2}+\log \alpha_{3} \\
& \leqq \log \left\{\max \left(\alpha_{2}, \beta_{2}\right)\right\}+\log \left\{\max \left(\alpha_{3}, \beta_{3}\right)\right\} \\
& =d(x, z)+d(z, y)
\end{aligned}
$$

and similarly if $\beta_{1} \geqq \alpha_{1}$.

Note that in [1] and [4] the metric is defined on the intersection of $K$ with a hyperplane and is, in fact, $d^{\prime}(x, y)=\log (\alpha \beta)$ which is equivalent to the above metric on such a section but does not define a metric on a constituent because $d^{\prime}(x, y)=0$ when $x=\lambda y$. We observe, also, that this construction only requires that $K$ be closed in some linear topology.

Lemma 3. Let $K$ be a normal cone which is complete in the norm topology, then each constituent, $C$, is complete with respect to $d($,$) .$

Proof. Let $\left\{x_{n}\right\}$ be a sequence in $C$ which is Cauchy with respect to $d($,$) and let \alpha_{p q}=\inf \left\{\lambda: x_{p} \leqq \lambda x_{q}\right\}(p, q=1,2, \cdots)$. It is proved first that $\left\{x_{n}\right\}$ is bounded in norm, secondly that it is a Cauchy sequence in norm and therefore converges to an element $u \in K$, and thirdly that the sequence converges to $u$ in the metric and that $u \in C$.

(i) Since $\left\{x_{n}\right\}$ is a Cauchy sequence there exists $N$ such that $d\left(x_{p}, x_{q}\right)<1$ for all $p, q \geqq N$, i.e., $\max \left(\alpha_{p q}, \alpha_{q p}\right)<\exp (1)(p, q \geqq N)$. In particular $\alpha_{p N}<\exp (1)(p \geqq N)$ so that $x_{p} \leqq \exp (1) x_{N} \leqq 3 x_{N}$. So, since $K$ is normal, $\left\|x_{p}\right\| \leqq 3 \gamma\left\|x_{N}\right\|$. Thus $\left\{\left\|x_{n}\right\|\right\}$ is bounded by $m$ where

$$
m=\max \left\{\left\|x_{1}\right\|, \cdots,\left\|x_{N}\right\|, 3 \gamma\left\|x_{N}\right\|\right\} .
$$

(ii) Given $\epsilon>0$ there exists $\delta>0$, depending on $\epsilon$, such that $\exp (\delta) \leqq 1+(\epsilon / M)$ where $M=m(1+2 \gamma)$. Again because $\left\{x_{n}\right\}$ is a Cauchy sequence there exists $N_{\epsilon}$ such that

$$
d\left(x_{p}, x_{q}\right)<\delta \quad\left(p, q \geqq N_{\epsilon}\right),
$$

i.e., $\max \left(\alpha_{p q}, \quad \alpha_{q p}\right)<1+(\epsilon / M)$. Thus $x_{p} \leqq(1+(\epsilon / M)) x_{q}$ and $x_{q}$ $\leqq(1+(\epsilon / M)) x_{p}$ and so, by Lemma 1 ,

$$
\left\|x_{p}-x_{q}\right\| \leqq m(1+2 \gamma)(1+(\epsilon / M)-1)=\epsilon \quad\left(p, q \geqq N_{\epsilon}\right) .
$$

Hence $\left\{x_{n}\right\}$ is a Cauchy sequence in norm and, since $K$ is complete, there exists an element $u \in K$ such that $\lim _{n \rightarrow \infty}\left\|x_{n}-u\right\|=0$.

(iii) As before, $d\left(x_{p}, x_{q}\right)<\epsilon$ for all sufficiently large $p$ and $q$, i.e., $x_{p} \leqq \exp (\epsilon) x_{q}$ and $x_{q} \leqq \exp (\epsilon) x_{p}$. 

fore

But $\lim _{q \rightarrow \infty}\left\|x_{q}-u\right\|=0$ and $K$ is closed in the norm topology there-

$$
x_{p} \leqq \exp (\epsilon) u \text { and } u \leqq \exp (\epsilon) x_{p}
$$

for all sufficiently large $p$. This implies that $u \in C$ (it is linked with $\left.x_{p}\right)$ and that $d\left(x_{p}, u\right) \leqq \epsilon$ for all large $p$ but since $\epsilon$ is chosen arbitrarily this is the same as saying that $\left\{x_{n}\right\}$ converges to $u$ with respect to the metric.

Remarks. 1. $X$ itself need not be complete. 2. This lemma and, consequently, the following theorem are true also for locally convex spaces when "normal" is given a suitable meaning. If $X$ is a vector space with a locally convex topology generated by a system of seminorms $\left\{p_{\alpha}\right\} K$ is said to be normal if, for each $\alpha$, there exists a positive real number $\gamma_{\alpha}$ such that $0 \leqq x \leqq y$ implies $p_{\alpha}(x) \leqq \gamma_{\alpha} p_{\alpha}(y)$. See Bonsall [2] and also Schaefer [5]. Then the lemma is proved by replacing $\|\cdot\|$ by $p_{\alpha}(\cdot)$ everywhere and "norm topology" by "locally convex topology."

\section{Contraction mapping.}

TheOREM. Let $K$ be a complete, normal cone and let $T$ be a mapping of $X$ into itself with the properties:

(i) there exists $p$ with $0 \leqq p<1$ such that $x, y \in K, x \leqq \alpha y$ and $y \leqq \beta x$

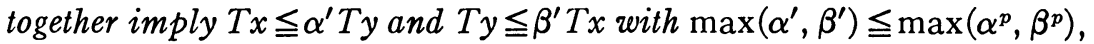

(ii) there exists an $x_{0}$ such that $x_{0}$ and T $x_{0}$ are linked.

Then there exists a vector $u$ belonging to the constituent containing $x_{0}$ such that $T u=u$ and this $u$ is unique in that constituent. Moreover, the iterative sequence defined by $x_{n}=T x_{n-1}(n=1,2, \cdots)$ converges in norm to $u$.

Proof. Let $C$ be the constituent containing $x_{0}$, then $T(C) \subseteq C$. For, let $y \in C$, then $x_{0}$ and $y$ are linked and hence, by property (i), so are $T x_{0}$ and $T y$, and therefore $x_{0}$ and $T y$ are linked (by property (ii) and the transitivity of the equivalence relation). This means that $T y \in C$ and shows, incidentally, that if (ii) is satisfied by one $x_{0}$ it is satisfied by all points in that constituent.

Evidently, from property (i), $T$ is a contraction of $C$ with respect to $d($,$) and C$ is complete in this metric by Lemma 3 so that the existence of a unique fixed point $u$ in $C$ follows from the contraction mapping principle. The fact that $\left\{x_{n}\right\}$ converges in norm follows from the fact that it is Cauchy in the metric and hence Cauchy in norm (from the proof of Lemma 3 ).

Corollary. Suppose $\lambda>0$, then there exists $u_{\lambda} \in C$ (which is unique in C) such that $T u_{\lambda}=\lambda u_{\lambda}$. 
Proof. Apply the theorem to the operator $T_{\lambda}=\lambda^{-1} T$.

Remarks. 1 . The vector $u$ belongs to $C$ and, therefore, is not zero.

2 . With the hypotheses of the theorem it is possible to prove without any overt appeal to the subsidiary metric that $\left\{x_{n}\right\}$ is Cauchy in norm, that its limit, $u$, is a fixed point of $T$ and that this $u$ is unique in the constituent containing $x_{0}$. However, the proof is, in effect, the proof of the contraction mapping principle.

3. Condition (i) on $T$ can be replaced by the slightly more restrictive but perhaps more natural criterion.

(i) ${ }^{\prime}$ There exists $p$ with $0 \leqq p<1$ such that $x \in K$ and $x \leqq \alpha y \Rightarrow T x$ $\leqq \alpha^{p} T y$.

4. It is not necessary to suppose that $T$ is positive (i.e., that it maps $K$ into itself) though, as has been seen, the assumptions do imply that $T$ maps any constituent of $K$ which contains an $x_{0}$ with property (ii) into itself.

5. (ii) is satisfied if, for example, some order unit $e$ is mapped into an order unit $e^{\prime}$.

6. The case $p=1$ is critical for this method, and, in general, extra conditions are needed to obtain results; this is true, in particular, for linear operators which map $K$ into itself. For example, Samelson's method, [4], will extend to infinite dimensional spaces if a restricted compactness condition is imposed on the operator while Birkhoff supposes in addition that $T$ is "uniformly positive" which condition is sufficient to prove that an operator related to $T$ is a contraction with respect to the metric $d^{\prime}($,$) mentioned above.$

\section{Examples.}

1. Let $X$ be the space $C[0,1]$ of continuous, real-valued functions on the unit interval and let $T$ be defined by the equation,

$$
(T f)(x)=\int_{0}^{1} k(x, y,|f(y)|) d y \quad(0 \leqq x \leqq 1)
$$

where $k(x, y, z)$ is a real-valued function of three variables which is a continuous function of $(x, y, z) \in[0,1]^{2} \times[0, \infty)$ and which has the properties:

( $\alpha) k\left(x, y, z_{1}\right) \leqq k\left(x, y, z_{2}\right)$ for $0 \leqq z_{1} \leqq z_{2}$ and all $x, y \in[0,1]$;

( $\beta$ ) there exists $p$ with $0 \leqq p<1$ and $k(x, y, \alpha z) \leqq \alpha^{p} k(x, y, z)$;

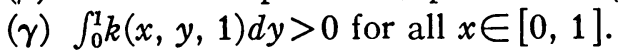

For example, let $k(x, y, z)$ be of the form

$$
\sum_{r=1}^{n} k_{r}(x, y) z^{p_{r}}
$$


where $k_{r}(x, y)$ are non-negative, continuous functions of $x$ and $y$ and $0 \leqq p_{r}<1(r=1, \cdots, n)$. Then $(\alpha)$ and $(\beta)$ together imply that $T$ has property (i)' while $(\gamma)$ ensures that the unit function is linked with its image under $T$. Thus the theorem is applicable to such an operator and there is a unique everywhere positive function which is a fixed point of $T$.

2. With the same space $X$ and with the same notation as in example 1 , let $p$ be an arbitrary positive number, then $T_{q}$ defined by

$$
\left(T_{q} f\right)(x)=\{(T f)(x)\}^{1 / q} \quad(q>p)
$$

has properties (i)' and (ii) and hence there exists a function $u_{q}$, everywhere positive, such that $T_{q} u_{q}=u_{q}$ or

$$
\left(T u_{q}\right)(x)=\left\{u_{q}(x)\right\}^{q}(x \in[0,1]) \quad(\text { all } q>p) .
$$

I wish to thank Professor F. F. Bonsall for his help and encouragement in the preparation of this paper.

\section{REFERENCES}

1. G. Birkhoff, Extensions of Jentzch's theorem, Trans. Amer. Math. Soc. 85 (1957), 219-227.

2. F. F. Bonsall, The decomposition of continuous linear functionals into non-negative components, Proc. Univ. Durham Philos. Soc. Ser. A 13 (1957), 6-11.

3. H. Busemann, The geometry of geodesics, Academic Press, New York, 1955.

4. H. Samelson, On the Perron-Frobenius theorem, Michigan Math. J. 4 (1957), $57-59$.

5. H. Schaefer, Halbgeordnete lokalkonvexe Vektorräume, Math. Ann. 135 (1958), $115-141$.

King's College, Durham University, Newcastle upon Tyne 